\title{
Variation of the Spectrum of Operators in Infinite Dimensional Spaces
}

\author{
Mohammed Yahdi \\ Department of Mathematics and Computer Science, Ursinus College, Collegeville, USA \\ Email: myahdi@ursinus.edu
}

Received July 25, 2013; revised August 26, 2013; accepted September 29, 2013

Copyright (C) 2013 Mohammed Yahdi. This is an open access article distributed under the Creative Commons Attribution License, which permits unrestricted use, distribution, and reproduction in any medium, provided the original work is properly cited.

\begin{abstract}
The paper investigates the variation of the spectrum of operators in infinite dimensional Banach spaces. Consider the space of bounded operators on a separable Banach space when equipped with the strong operator topology, and the Polish space of compact subsets of the closed unit disc of the complex plane when equipped with the Hausdorff topology. Then, it is shown that the unit spectrum function is Borel from the space of bounded operators into the Polish space of compact subsets of the closed unit disc. Alternative results are given when other topologies are used.
\end{abstract}

Keywords: Operator Spectrum; Borel Function; Banach Space; Polish Space

\section{Introduction}

Let $X$ be an infinite dimensional Banach space. We denote by $T$ an arbitrary bounded operator on $X$ and by $I$ the identity operator on $X$. Let $\mathbb{D}$ be the closed unit disc of the complex plane $\mathbb{C}$. The restriction on $\mathbb{D}$ of the spectrum of an operator $T$, denoted by $\sigma(T)$, is the unit spectrum defined as follows:

$$
\sigma(T):=\{\lambda \in \mathbb{D} ;(T-\lambda I) \text { is not invertible }\} .
$$

Essential spectra of some matrix operators on Banach spaces (see [1]) and spectra of some block operator matrices (see [2]) were investigated, with applications to differential and transport operators. This paper investigates the variations of the unit spectrum $\sigma(T)$ as $T$ varies over the space $L(X)$ of all bounded operator on the Banach space $X$. First, we introduce the sets and the topologies required for this study.

\section{Definition 1}

- $\mathcal{K}(\mathbb{D})$ the set of all compact subsets of the closed unit disc $\mathbb{D}$ of the complex plane $\mathbb{C}$;

- $\sigma$ the spectrum function defined from $L(X)$ into $\mathcal{K}(\mathbb{D})$ that maps an operator $T$ to its unit spectrum $\sigma(T)$.

The set $\mathcal{K}(\mathbb{D})$ is endowed with the Hausdorff topology generated by the families of all subsets in one of the following forms

$$
\{F \in \mathcal{K}(\mathbb{D}) ; F \cap V \neq \varnothing\}
$$

and

$$
\{F \in \mathcal{K}(\mathbb{D}) ; F \subseteq V\},
$$

for $V$ an open subset of $\mathbb{D}$. Therefore, $\mathcal{K}(\mathbb{D})$ is a Polish space, i.e., a separable metrizable complete space, since $\mathbb{D}$ is Polish (see [3-5]). It is shown below that we can reduce the families that generate the above Hausdorff topology.

Proposition 1 Let $\mathcal{K}(\mathbb{D})$ be the set of compact subsets of the closed unit disc $\mathbb{D}$. Then $\mathcal{K}(\mathbb{D})$ equipped with the Hausdorff topology is a Polish space; where the Borel structure is generated by one of the following two families

$$
\{\{K \in \mathcal{K}(\mathbb{D}): K \cap V \neq \varnothing\} ; V \text { open in } \mathbb{D}\}
$$

and

$$
\{\{K \in \mathcal{K}(\mathbb{D}): K \subset V\} ; V \text { open in } \mathbb{D}\} .
$$

Proof 1 Let $V$ be an open subset of $D$. There exists a decreasing sequence of open subsets $\left(O_{n}\right)_{n \in \mathbb{N}}$; for example

$$
O_{n}=\left\{x \in \mathbb{D}: \operatorname{dist}\left(x, V^{c}\right) \leq \frac{1}{n}\right\},
$$

such that

$$
V^{c}=\bigcap_{n \in \mathbb{N}} O_{n} .
$$

We have

$$
\begin{aligned}
\{K \in \mathcal{K}(\mathbb{D}): K \cap V \neq \varnothing\}^{c} & =\left\{K \in \mathcal{K}(\mathbb{D}): K \subseteq V^{c}\right\} \\
& =\bigcap_{n \in \mathbb{N}}\left\{K \in \mathcal{K}(\mathbb{D}): K \subseteq O_{n}\right\} .
\end{aligned}
$$


On the other hand,

$$
\begin{aligned}
& \{K \in \mathcal{K}(\mathbb{D}): K \subseteq V\}^{c} \\
& =\left\{K \in \mathcal{K}(\mathbb{D}): K \cap V^{c} \neq \varnothing\right\} \\
& =\left\{K \in \mathcal{K}(\mathbb{D}): K \cap O_{n} \neq \varnothing, \forall n \in \mathbb{N}\right\} \\
& =\bigcap_{n \in \mathbb{N}}\left\{K \in \mathcal{K}(\mathbb{D}): K \cap O_{n} \neq \varnothing\right\} .
\end{aligned}
$$

Indeed, if for all $n \in \mathbb{N}$, there exists $x_{n} \in K \cap O_{n}$, then there exists a subsequence $\left(x_{n_{k}}\right)_{k}$ of $\left(x_{n}\right)_{n}$ that converges to $x \in K$, and since $\left(O_{n}\right)_{n}$ is decreasing, we have

$$
x \in \bigcap_{n} O_{n} .
$$

\section{Norm Operator Topology and the Spectrum Function}

We equip $L(X)$ with the canonical norm of operators defined by

$$
\|T\|=\sup _{x \in B_{X}}\|T(x)\| \text {, where } B_{X} \text { is the unit ball of } X \text {. }
$$

Note that the map $\sigma: T \mapsto \sigma(T)$ is not continuous when $L(X)$ is endowed with its canonical norm. Indeed, the operators $T_{n}=\left(1+\frac{1}{n}\right) I$ converge to the identity $I$ while $\sigma\left(T_{n}\right)=\varnothing$ and $\sigma(I)=\{1\}$. However, we have the following result.

Proposition 2 Let $X$ be a Banach space, $(L(X),\|\|$. the space of bounded operators equipped with the norm of operators, and $\mathcal{K}(\mathbb{D})$ the set of compact subsets of the unit disc $\mathbb{D}$ equipped with the Hausdorff topology. Then the spectrum map

$$
\begin{aligned}
\sigma & :(L(X),\|.\|) \rightarrow \mathcal{K}(\mathbb{D}) \\
T & \mapsto \sigma(T)
\end{aligned}
$$

is upper-semi continuous.

Proof 2 Let $V$ be an open subset of $\mathbb{D}$. By proposition 1 , it is only needed to show that the set

$$
O_{V}=\{T \in L(X) ; \sigma(T) \subseteq V\}
$$

is $\|$.$\| -open in L(X)$. Let $T_{0}$ be fixed in $O_{V}$. Since $\sigma\left(T_{0}\right) \cap \mathbb{D} \subseteq V$, then for all $\lambda \in \mathbb{D} \backslash V$,

- The operator $\left(T_{0}-\lambda I\right)$ is invertible;

- And the map $\lambda \in \mathbb{D} \backslash V \mapsto\left(T_{0}-\lambda I\right)^{-1}$ is continuous (see [6]).

It follows that

$$
\sup _{\lambda \in \mathbb{D} \backslash V}\left\|(T-\lambda I)^{-1}\right\|<+\infty
$$

since $\mathbb{D} \backslash V$ is compact. Put

$$
\delta=\inf _{\lambda \in \mathbb{D} \backslash V} \frac{1}{\left\|\left(T_{0}-\lambda I\right)^{-1}\right\|}>0 .
$$

Let $T \in L(X)$ such that $\left\|T-T_{0}\right\|<\delta$.

For any $\lambda \in \mathbb{D} \backslash V$ we have

$$
\left\|(T-\lambda I)-\left(T_{0}-\lambda I\right)\right\|=\left\|T-T_{0}\right\|<\frac{1}{\left\|\left(T_{0}-\lambda I\right)^{-1}\right\|} .
$$

Thus, $(T-\lambda I)$ is invertible and hence $\lambda \notin \sigma(T)$. In other terms, $\sigma(T) \subseteq V$ for all $T \in L(X)$ with $\left\|T-T_{0}\right\|$ $<\delta$. Therefore $O_{V}$ is an open subset of $(L(X),\|\|$.$) .$

\section{Strong Operator Topology and the Spectrum Function}

Consider now $L(X)$ equipped with the strong operator topology $S_{o p}$ (see [6]). In general, $L(X)$ equipped with the strong operator topology is not a polish space (since it is not a Baire space). However, if $X$ is separable, then $\left(L(x), S_{o p}\right)$ is a standard Borel space. Indeed, it is Borel-isomorph to a Borel subset of the Polish space $X^{\mathbb{N}}$ equipped with the norm product topology via the map

$$
\begin{aligned}
& \varphi:\left(L(X), S_{o p}\right) \rightarrow\left(X^{\mathbb{N}}, \mathcal{P}\right) \\
& T \mapsto\left(T z_{n}\right)_{n \in \mathbb{N}},
\end{aligned}
$$

where $\left\{z_{n}, n \in \mathbb{N}\right\}$ is a dense $\mathbb{Q}$-vector space in $X$.

The next result shows how this topology on $L(x)$ affects the spectrum function.

Theorem 1 For any separable infinite dimensional Banach $X$, the map

$$
\begin{aligned}
\sigma: L(X) \rightarrow \mathcal{K}(\mathbb{D}) \\
T \mapsto \sigma(T),
\end{aligned}
$$

which maps a bounded operator to its unit spectrum, is Borel when $L(X)$ is endowed with the strong operator topology $S_{o p}$ and $\mathcal{K}(\mathbb{D})$ with the Hausddorf topology.

Proof 3 As $\mathcal{K}(\mathbb{D})$ is equipped with the Hausdorff topology, it follows from the proposition 1, that it is enough to show that for any open subset $V$ of the disc $\mathbb{D}$, the following subset $E_{V}$ is Borel in $\left(L(X), S_{\text {op }}\right)$

$$
E_{V}=\{T \in L(X): \sigma(T) \cap V \neq \varnothing\} .
$$

Let $V$ be a fixed open subset of $\mathbb{D}$. We have

$$
E_{V}=P_{L(X)}(\Omega),
$$

where $P_{L(X)}$ stands for the canonical projection of $L(X) \times \mathbb{D}$ onto $L(X)$, and

$$
\Omega=\{(T, \lambda) \in L(X) \times V: \lambda \in \sigma(T)\} .
$$

By a descriptive set theory result from ([7]), to show 
that $E_{V}$ is a Borel set it suffices to show that $\Omega$ is a Borel set with $K_{\sigma}$ vertical sections.

For $T \in L(X)$, the vertical section of the set $\Omega \subseteq L(X) \times \mathbb{D}$ along the direction $T$ is given by

$$
\begin{aligned}
\Omega(T) & =\{\lambda \in \mathbb{D}:(T, \lambda) \in \Omega\} \\
& =\{\lambda \in \mathbb{D}: \lambda \in V \cap \sigma(T)\} \\
& =\sigma(T) \cap V .
\end{aligned}
$$

Thus, it is indeed a $K_{\sigma}$ of $\mathbb{D}$.

Now, we need to prove that $\Omega$ is a Borel set. Put

$$
\Delta=\{(T, \lambda) \in L(X) \times \mathbb{D}: \lambda \in \sigma(T)\} .
$$

Therefore

$$
\Omega=\Delta \cap L(X) \times V,
$$

Hence, to finish the proof, it is enough to prove the following claim.

Claim: $\Delta$ is a Borel set of $L(X) \times \mathbb{D}$.

First, note that $\Delta=A \cup B$ with

$$
\begin{aligned}
A=\{ & (T, \lambda) \in L(X) \times \mathbb{D}: T-\lambda I \\
& \text { is not isomorph to its range }\}, \\
B=\{ & (T, \lambda) \in L(X) \times \mathbb{D}:(T-\lambda I)(X) \\
& \text { is not dense in } X\} .
\end{aligned}
$$

Indeed, if $(T-\lambda I)$ is an isomorphism onto its range, then $(T-\lambda I)(X)$ is a closed subspace that will be strict if $\lambda \in \sigma(T)$, and thus not dense in $X$. On the other hand, since $X$ is separable, there exists a countable and dense subset $\mathcal{Y}$ in the sphere $S_{X}$ of $X$, and there exists a dense sequence $\left\{x_{n}\right\}_{n \in \mathbb{N}}$ in $X$.

Now, we will show that $A$ and $B$ are Borel sets. Let $(T, \lambda) \in L(X) \times \mathbb{D}$. From the definition of $A$, We have $(T, \lambda) \in A$ if and only if

$$
\exists\left(z_{n}\right)_{n \in \mathbb{N}} \subseteq S_{X}: \lim _{n \rightarrow \infty}\left\|(T-\lambda I) z_{n}\right\|=0 .
$$

In other terms, this is equivalent to

$$
\begin{aligned}
& \exists\left(z_{n}\right)_{n \in \mathbb{N}} \subseteq \mathcal{Y}, \forall k \geq 1 \exists N_{k} \in \mathbb{N} \\
& \forall n \geq N_{k}:\left\|(T-\lambda I) z_{n}\right\|<\frac{1}{k} .
\end{aligned}
$$

By choosing the subsequence $\left(z_{N_{k}}\right)_{k \in \mathbb{N}}$ instead of $\left(z_{n}\right)_{n \in \mathbb{N}}$, the previous statement is equivalent to

$$
\begin{aligned}
& \exists\left(z_{n}\right)_{n \in \mathbb{N}} \subseteq \mathcal{Y}, \forall k \geq 1 \\
& \exists N_{k} \in \mathbb{N}:\left\|(T-\lambda I) z_{N_{k}}\right\|<\frac{1}{k},
\end{aligned}
$$

or again,

$$
\forall k \geq 1, \exists x \in \mathcal{Y}:\|T x-\lambda x\|<\frac{1}{k}
$$

Therefore,

$$
A=\bigcap \bigcup_{k \geq 1 x \in \mathcal{Y}} A_{k}^{x}
$$

with

$$
A_{k}^{x}=\left\{(T, \lambda) \in L(X) \times \mathbb{D}:\|T x-\lambda x\|<\frac{1}{k}\right\} .
$$

Since $L(X)$ is equipped with the the strong operator convergence $S_{o p}$, it follows that the sets $A_{k}^{X}$ are open. Hence, $A$ is a Borel set.

On the other hand, " $(T-\lambda I)(X)$ is not dense in $X$ " is equivalent to

$$
\begin{aligned}
& \exists y \in S_{X} \text { and } \exists k \geq 1 \text { such that } \\
& \forall x \in X:\|y-(T-\lambda I) x\| \geq \frac{1}{k},
\end{aligned}
$$

or again,

$$
\begin{aligned}
& \exists y \in \mathcal{Y} \text { and } \exists k \geq 1 \text { such that } \\
& \forall n \in \mathbb{N}:\left\|y-(T-\lambda I) x_{n}\right\| \geq \frac{1}{k} .
\end{aligned}
$$

Therefore

$$
B=\bigcup_{y \in \mathcal{Y}} \bigcup_{k \in \mathbb{N} n \in \mathbb{N}} \bigcap_{k, n}^{y}
$$

with

$$
B_{k, n}^{y}=\left\{(T, \lambda) \in L(X) \times \mathbb{D} ;\left\|y-(T-\lambda I) x_{n}\right\| \geq \frac{1}{k}\right\} .
$$

Similarly to $A_{k}^{x}$, it is not difficult to see that the sets $B_{k, n}^{y}$ are Borel sets. Hence $B$ is also a Borel set. This proves the claim and ends the proof of the theorem 1 .

\section{Conclusions}

The variation of the unit spectrum of operators in infinite dimensional Banach spaces is investigated. The unit spectrum of an operator $T$, denoted by $\sigma(T)$, is defined as the restriction on the closed unit disc $\mathbb{D}$ of the complex plane $\mathbb{C}$ of the spectrum of $T$ given by $\{\lambda:(T-\lambda I)$ is not invertible $\}$.

First, the paper presents a simplified characterization of the Borel structure making the set $\mathcal{K}(\mathbb{D})$ of compact subsets of the closed unit disc $\mathbb{D}$ a Polish space. It is also shown that for a Banach space $X$, the map $\sigma: T \in L(X)$ that for an operator associates its unit spectrum $\sigma(T) \in \mathcal{K}(\mathbb{D})$ is upper-semi continuous when $L(X)$ is endowed with the norm of operators. On the other hand, when $L(X)$ is endowed with the strong operator topology, it is shown that first $X$ needed to be a separable infinite dimensional Banach to guarantee a standard Borel structure on $L(X)$, then it is shown that the that the map $\sigma: T \in L(X)$ is Borel in this case. Therefore, this topology is making the spectrum function more rigourous, and as a consequence the variations of the spectrum following changes in an operator or a se- 
quence of operators.

\section{REFERENCES}

[1] M. Damak and A. Jeribi, "On the Essential Spectra of Matrix Operators and Applications,” Electronic Journal of Differential Equations, Vol. 2007, No. 11, 2007, pp. 1-16.

[2] A. Jeribia, N. Moalla and I. Walhaa, "Spectra of Some Block Operator Matrices and Application to Transport Operators,” Doklady Akademii Nauk SSSR (N.S.), Vol. 351, No. 1, 2009, pp. 315-325.

[3] A.S. Kechris and A. Louveau, "Descriptive Set Theory and the Structure of Sets of Uniqueness," Cambridge
University Press, Cambridge, 1987.

[4] K. Kuratowski, “Topology,” Vol. II, Academic Press New York, 1966.

[5] J. P. R. Christensen, "Topology and Borel Structure," North-Holland Mathematics Studies, Vol. 10, Elsevier, Amsterdam, 1974.

[6] N. Dunford and J. Schwartz, "Linear Operator," Part. I, DA Wiley-Interscience Publication, New York, London, Sydney, 1971.

[7] J. Saint Raymond, “Boréliens à Coupes $K_{\sigma}$, , Bulletin de la Société Mathématique de France, Vol. 104, 1976, pp. 389-400. 